\title{
Linking multi-level population dynamics: trait, role, and population
}

\author{
Nao Takashina ${ }^{1}$ \\ ${ }^{1}$ The University of Tokyo
}

December 2, 2020

\begin{abstract}
Species interactions characterize population dynamics and ecosystem structure. While the population-level discussion is common in many ecological studies, trait variations within a population and ontogenetic diet/trophic niche shift are prevail across taxa. The ontogenetic development may lead to an individual's role shift, such as inferior/superior competitor, prey, or predator. Here, we develop a novel mathematical framework to bridge multiple levels of population dynamics, such as trait, role, and population-level. We start with a nonlinear trait-level model, and derive role-level and population-level dynamics. By utilizing the connections, we demonstrate that the population-level model predicts the equilibrium status of the role-level model. In the role-level model, we discuss multiple role-shift scenarios: from (i) inferior/superior competitor to superior/inferior competitor, (ii) competitor to predator, and (iii) prey to predator. Our approach connects traits, roles, and population dynamics consistently, thus offering an opportunity to discuss the effect of species traits in the population-level dynamics.
\end{abstract}

\section{Hosted file}

Main.pdf available at https://authorea.com/users/380879/articles/496667-linking-multi-levelpopulation-dynamics-trait-role-and-population 